\title{
Prisoner's dilemma in structured scale-free networks
}

\author{
Xing $\mathbf{L i}^{1,2}$, Yonghui $\mathbf{W u}^{1,2}$, Zhihai Rong ${ }^{3}$, Zhongzhi Zhang ${ }^{1,2}$, \\ Shuigeng Zhou $^{1,2}$ \\ 1 School of Computer Science, Fudan University, Shanghai 200433, China \\ 2 Shanghai Key Lab of Intelligent Information Processing, Fudan University, \\ Shanghai 200433, China \\ ${ }^{3}$ Department of Automation, Donghua University, Shanghai 201620, China \\ E-mail: yhwu@fudan.edu.cn, zhangzz@fudan.edu.cn, sgzhou@fudan.edu.cn
}

\begin{abstract}
The conventional wisdom is that scale-free networks are prone to cooperation spreading. In this paper we investigate the cooperative behaviors on the structured scale-free network. On the contrary of the conventional wisdom that scale-free networks are prone to cooperation spreading, the evolution of cooperation is inhibited on the structured scale-free network while performing the prisoner's dilemma (PD) game. Firstly, we demonstrate that neither the scale-free property nor the high clustering coefficient is responsible for the inhibition of cooperation spreading on the structured scale-free network. Then we provide one heuristic method to argue that the lack of age correlations and its associated 'large-world' behavior in the structured scale-free network inhibit the spread of cooperation. The findings may help enlighten further studies on evolutionary dynamics of the PD game in scale-free networks.
\end{abstract}

PACS numbers: 87.23.Kg, 89.75.Hc, 87.23.Kg, 02.50.Le 


\section{Introduction}

Cooperation is an essential factor for the evolution of species. For decades, scientists have been embarking on the problem of understanding the emergence of cooperation [1, 2, 3, 4, 5, 6]. In the field of this investigation, evolution game theory has become a powerful tool [7, 8, 9]. One of the most frequently used metaphors is the prisoner's dilemma (PD) game [10] that is modeled to simplify individual interactions when selfish actions provide a short-term higher payoff. In each round two players are involved in the game. The two strategies for one player are to become a cooperator or a defector. A cooperator is someone who pays some cost for another individual to receive a benefit. A defector has no cost and does not deal out benefits. Cost and benefit are measured in terms of fitness. The combinations of the strategies will be of great difference. When both of the players choose to cooperate or to defect, each of the two will get benefit $R$ or $P$, respectively. When one player choose to cooperate and the other choose to defect, the cooperator will get $S$, while the defector will get $T$. In the PD game, the order for the payoffs with different combinations of strategies is $T>R>P>S$. So the best strategy is to defect regardless the opponent's strategy and assuming that strategies are allowed to spread within the population according to their payoffs [7].

Recently, biological experiments have demonstrated that the evolution dynamics is related to the topological structure of the media, on top of which the evolution dynamics are performed [11]. Earlier work [2, 4, 12, 13] mainly focused on the twodimensional lattice, which is modeled for the homogeneous network. In these studies, a player is constrained to play solely with its nearest neighbors. All the studies reported that unlike in unstructured populations, cooperators and defectors can coexist in the lattice indefinitely. However, the regular network is not suitable for modeling real networks since a lot of empirical studies [14, 15, 16, 17] have uncovered that the degree distribution $P(k)$ of many real-life systems complies with a power-law form $P(k) \sim k^{-\lambda}$ with $2<\lambda \leqslant 3$. The scale-free property shows that everyone plays a different role in the network, which describes a kind of social diversity [18]. A famous model for generating scale-free network is the Barabási-Albert (BA) model [19].

The evolution of cooperation on scale-free networks has been explored in [20, 21, 22, 23, 24, 25], which exhibited that cooperation is the dominating trait throughout the entire range of parameters. In this paper, we focus on the evolution of cooperation on a structured scale-free network, which demonstrates negative age-correlations [26]. Firstly, we introduce the algorithm for constructing the network. Secondly, we simulate the PD game on the network, and compare the results with that of the BA network. In contrast to the results previously obtained for the BA network, we find that the structured scale-free network inhibits the spread of cooperation. Finally, we provide a heuristic argument to explain why the spread of cooperation is inhibited on the network considered, which is justified by extensive simulations. 


\section{Models}

Firstly let us introduce the celebrated BA model: Initially there are $m_{0}$ connected nodes in the network. At each time step, one node is added into the network and links with $m\left(m<m_{0}\right)$ existing nodes. The probability that an existing node $i$ acquires a new link is $P\left(k_{i}\right)=\frac{k_{i}}{\sum_{j} k_{j}}$, where $k_{i}$ is the degree of node $i$. Repeat this step until getting the desired network size. In the BA model, on one hand, the chance that an old node receiving a new link is proportional to it age; this phenomenon is called "preferential attachment", also named "age-correlations". On the other hand, the longrange connections generated by the process decrease the distance between the vertices, leading to a small-world phenomenon: the average path length increases logarithmically with the network size [27].

The aging of nodes is particularly interesting. Recently, Klemm and Eguíluz found that for the scientific citation network [16], the age-correlation is negative: the mean citation rate of papers decreases with the increase of their age [26], that is to say, old nodes have less probability to obtain links than those nodes just added into the network. To describe this phenomenon, they put forward a new model called the structured scalefree network (also called highly clustered scale-free network). Their model is built as follows: Initially there is a complete graph with $m$ active nodes. At successive time step, one active node is added into the network connected to all $m$ active nodes, then one of $m+1$ active nodes is randomly chosen and deactivated with probability $P\left(k_{j}\right)=\frac{s}{a+k_{j}}$ with $a>0$ being a constant, where $s$ is a normalization factor defined by $s^{-1}=\sum_{i} \frac{1}{a+k_{i}}$, in which $i$ belongs to the set of active nodes. Repeat the process until the network size reaches the needed size.

According to the above process, we know the age correlations are greatly suppressed since every round only the active nodes, not all existing nodes, have the chance to acquire new links. In result, the linear topology of the structured scale-free network with local highly connected clusters forms a long chain [28]. The power law exponent is $\lambda=2+\frac{a}{m}$. In this paper, we take $a=m$, and thus the structured scale-free network has the same degree distribution with the BA model with $\lambda=3$ [19]. The clustering coefficient of BA network approaches to zero as network size grows to infinite, while in the structured scale-free network it is an asymptotic value $\frac{5}{6}$ for the case $a=m$.

After introducing the two models, we will study the evolutionary PD game on both models in the following section.

\section{Simulations}

In this section, we implement the finite population analogue of replicator dynamics in the following PD simulations. Following [2], we make $T=b>1, R=1$, and $P=S=0$ in the PD game, where $b$ represents the temptation to defect, being typically constrained to the interval $1<b \leqslant 2$. In each round of evolution, one node $i$ plays the PD game with its directly connected neighbors, accumulating the payoff as $P_{i}$. 
Whenever the strategy of the player $i$ with $k_{i}$ neighbors is to be updated, a neighbor $j$ is randomly selected from $i$ 's neighborhood. If $P_{j}>P_{i}$, the chosen neighbor $j$ spreads

its strategy to the player $i$ with probability $\frac{P_{j}-P_{i}}{b\left(\max \left(k_{i}, k_{j}\right)\right)}$, otherwise, the player $i$ holds its strategy [20]. Simulations were carried out for a population with $N=10000$ individuals. Initially, cooperator and defector strategies were distributed randomly among the players. Equilibrium frequencies of cooperators and defectors were obtained by averaging over 1000 generations after a transient time of 50000 generations. The frequency of cooperators is a function of the parameter $b$ for the PD game. Each data point corresponds to 100 simulations that are 10 runs for 10 different realizations of the same type of network specified by the appropriate parameters (the population size $N$ and the average connectivity $z$ ).

Figure 1 shows the results for the PD game on both networks with different values of the average connectivity $z$. The BA scale-free network and the structured scale-free network are constructed through above methods. The frequency of cooperators, $f_{c}$, is enhanced on the BA scale-free networks in the PD game, dominating over the entire range of $b(1 \leqslant b \leqslant 2)$. Notice that even when $2<b \leqslant 3$, we have checked that the results will not change. When $1<b \leqslant 1.4$, both of the networks are all-cooperator networks. As the temptation value $b$ increases from 1.4, the frequency of cooperators in the structured scale-free network descends rapidly while the cooperative behavior is always blooming in the BA network. Especially, for $b=2$, when $z=4, f_{c}$ on the structured scale-free network is just half of that on the BA network; when $z=8, f_{c}$ decreases to 0 on the structured scale-free network, while the BA network still shows a remarkable survival of cooperation with $f_{c} \approx 0.4$. On the other hand, as $z$ grows, the advantage of scale-free network shrinks. Therefore, it can be observed from figure 1 that cooperators change into defectors more quickly with smaller temptation value in the structured scale-free network, therefore, we can conclude that comparing with the BA network, the structured scale-free network decreases the frequency of cooperation, the reason for which will be detailedly analyzed in the next section.

\section{Analysis}

Why does the structured scale-free network inhibit the frequency of cooperators comparing with BA network? Firstly, we are aware of that both of the BA network and the structured scale-free network have the same power law exponent $(\lambda=3)$ in the above simulations. So the scale-free property is a trivial factor that can not affect the simulation results. Secondly, the clustering coefficient on the structured scale-free network is much higher than that in BA network. Assenza et al. in [29] find that high clustering coefficient can help the enhancement of cooperation on the scale-free networks with a tunable value of clustering coefficient [30], which is a different network model with the structured scale-free network. However, the high coefficient can not help to enhance the cooperation spreading on the structured scale-free network. So what is the root cause responsible for the inhibition of cooperation spreading on the structured scale- 


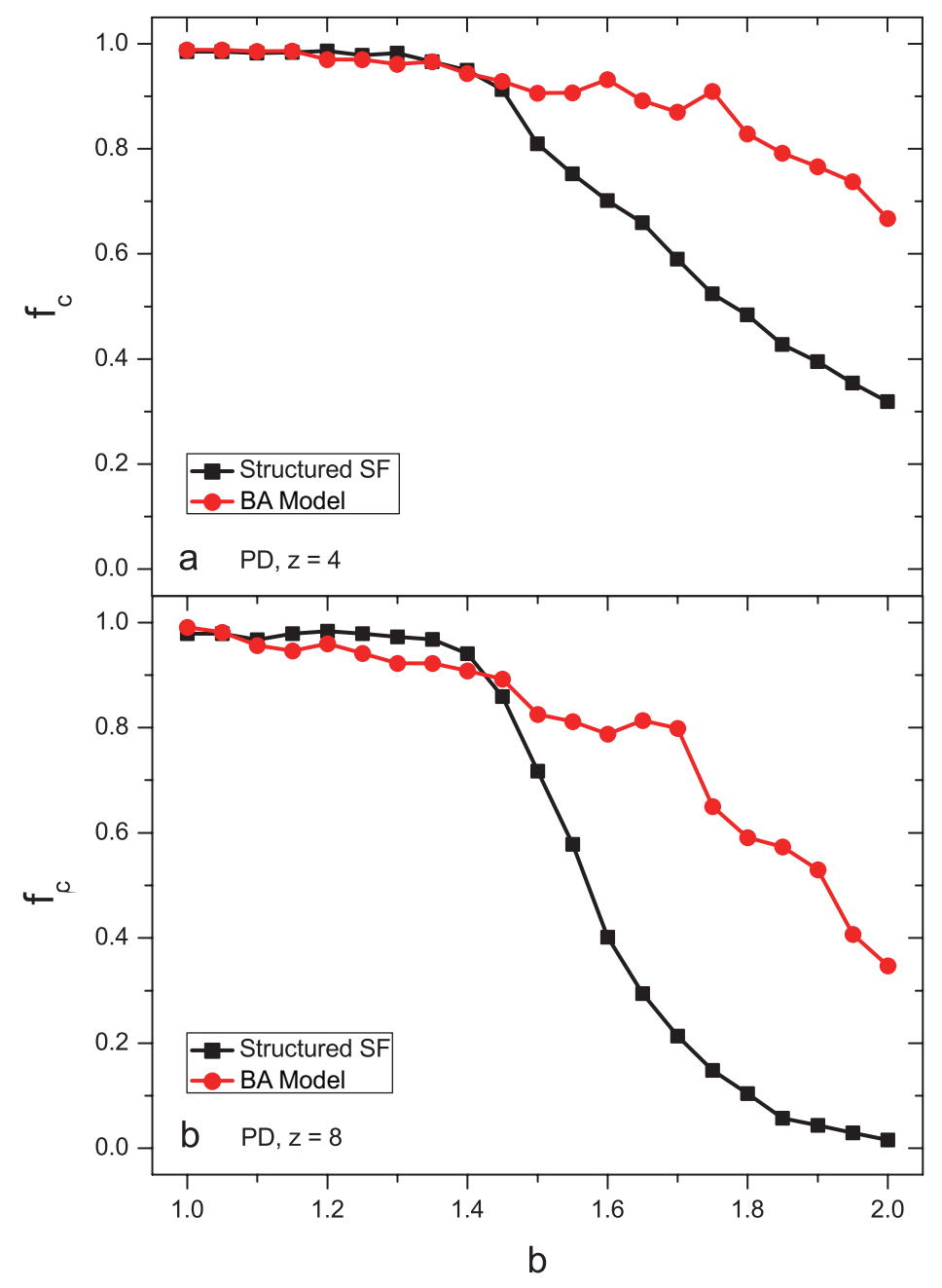

Figure 1. Frequency of cooperators $f_{c}$ as a function of the advantage $b$ of defectors for the PD game. The lines with filled squares show the results of structured scale-free network in the PD game. The lines with dots are for the BA scale-free network. In the figure, $z$ is the average connectivity of the network. According the construction process of the structured scale-free network, we know $z=2 \mathrm{~m}$. The frequency of cooperation in the structured scale-free network is surpassed by that of the BA network in almost the entire range of $b$, especially when $b$ is large enough.

free network? From the models, we know the age correlations are greatly suppressed on the structured scale-free network comparing with the BA network, which results in the fact that, in the BA network, a hub is usually linked to other highly connected nodes, while in the structured scale-free network, a hub is almost exclusively connected to low degree nodes [31. Also the diameter of the structured scale-free network is increasing linearly with the network size [28].

We can illustrate this phenomena through calculating the node-distance for one hub to others in the scale-free network as shown in figure 2. The value of node-distance from the origin to the destination is defined as the number of nodes on the shortest path between the two nodes. For example, the node-distance of a pair of directly connected 


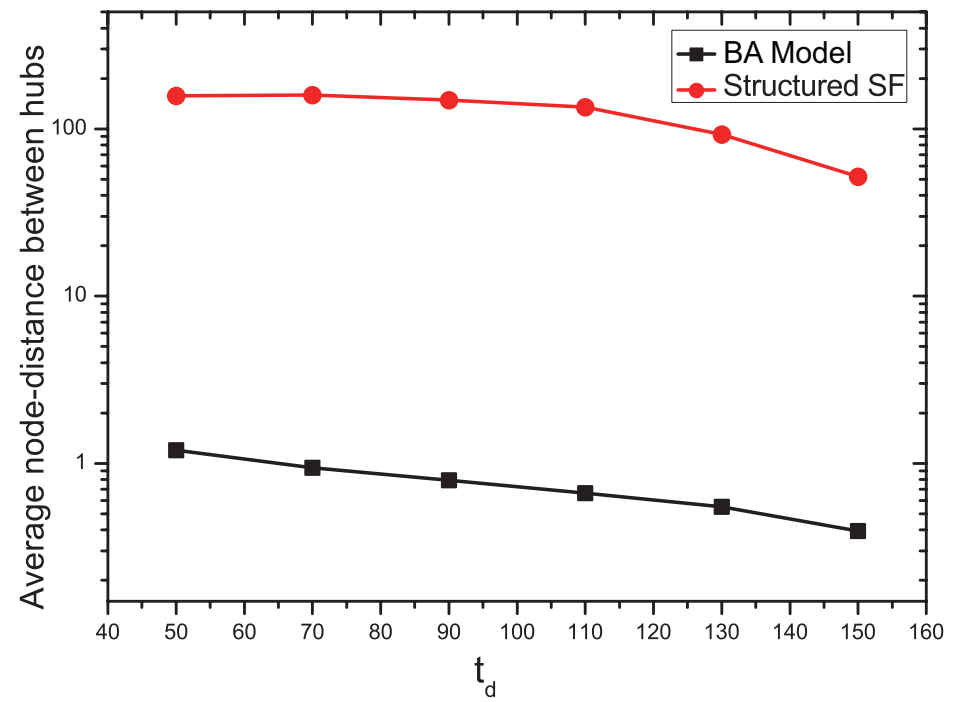

Figure 2. The average node-distance between hubs as a function of the threshold of degree, $t_{d}$. A node $i$ is called a hub node when its degree $k_{i} \geqslant t_{d}$. The solid line with dot symbols is for the structured scale-free network and the solid line with squares is for the BA network.

nodes is 0 ; if one of the shortest paths between nodes $i$ and $j$ bypass another node $k$, then the node-distance between $i$ and $j$ is 1, and so on. Figure 2 shows the average node-distance between hubs as a function of threshold $t_{d}$. A node $i$ can be qualified as a hub node only if its degree $k_{i} \geqslant t_{d}$. We can see that in the BA network, the hub nodes are usually interconnected with shorter average node-distance. While in the structured scale-free network, the average node-distance between hubs is rather high. When $t_{d}=50$, the node-distance among hubs on the structured scale-free network is more than 100, while this value is just a slightly more than one in the BA network. Hence, in the structured scale-free network, the hubs are connected through some low degree nodes, which form an "intermediate region".

For the sake of simplicity, we model the two kinds of network through two kinds of subgraph shown in figure 3, where (a) shows a directly connected double-star for the BA scale-free network and (b) is a double-star with intermediate region for the structured scale-free network. In figure 3(a), without losing generality, we assume that initially there are the unique defector locating on the right hub $h_{r}$ and others(including the left hub $h_{l}$ ) are cooperators. Set $T=b, R=1$, and $P=S=0$. In the first generation of the networked PD game, the payoff of $h_{l}$ is $\pi\left(h_{l}\right)=N$ and the payoff of $h_{r}$ is $\pi\left(h_{r}\right)=M b$. According to the evolutionary rule, the probability for the defector hub to invade the cooperator hub is

$$
P_{1}=\frac{\pi\left(h_{r}\right)-\pi\left(h_{l}\right)}{b \max (M, N)}=\frac{M b-N}{b \max (M, N)} .
$$

Moreover, the payoff of some leaf on the right star $l_{r}$ is $\pi\left(l_{r}\right)=0$ and the defector hub can also invade its leaves. The probability for the defector hub to invade its leaf $l_{r}$ 


\section{Cooperator $\triangle$ Defector}

(a)

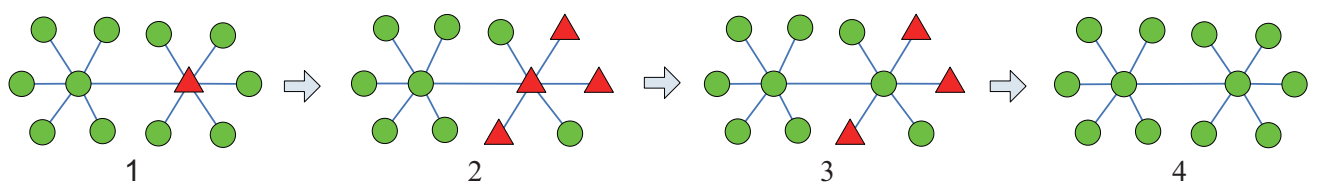

(b)

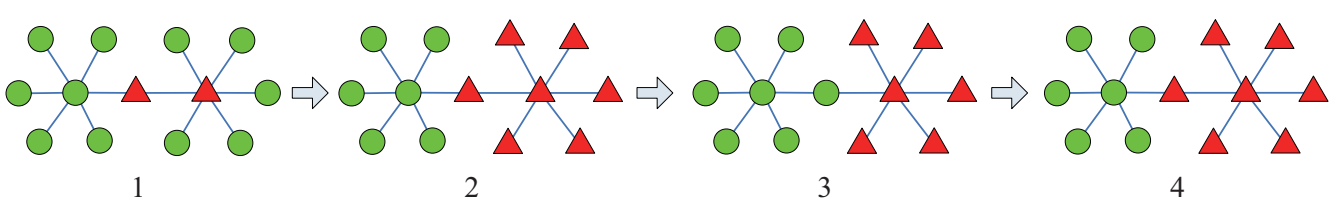

Figure 3. Evolution process on double-star graphs. (a) Directly connected doublestar for the BA network. The centers of the two stars are directly connected. The left hub $h_{l}$ has $N-1$ leaves and the right one $h_{r}$ has $M-1$ leaves. (b) Double-star with intermediate region for the structured scale-free network. Comparing with (a), the hubs are not directly connected, but by the intermediate region. The degrees of hubs are the same with (a). In this figure, we just put one node on the intermediate region for the sake of simplifying analysis.

is

$$
P_{2}=\frac{\pi\left(h_{r}\right)-\pi\left(l_{r}\right)}{b \max (1, M)}=1
$$

Apparently, $P_{1}<P_{2}$, hence, the leaves aside the defector hub are easier to be invaded by the defection than the cooperative hub. After the defector hub has invaded some of its leaves and $k(k<M)$ cooperator leaves survive, we get figure 3(a-2) where $\pi\left(h_{r}\right)=k b$. The probability that the defector hub invades the cooperator hub changes into

$$
P_{3}=\frac{\pi\left(h_{r}\right)-\pi\left(h_{l}\right)}{b \max (M, N)}=\frac{k b-N}{b \max (M, N)}
$$

Comparing with $P_{1}$, it can be found that the cooperator hubs are harder to be invaded by the defector hubs as the decrease of $k$. When $k=0$, i.e., all cooperator leaves aside the right star adopt the defector strategy, $P_{3}$ reaches its minimal value and the defector hub will definitely be invaded by the cooperator hub. Hence, after $h_{l}$ changes into the cooperator, it will induce its neighbors learning its behavior and the cooperative strategy will spread to the whole double-star graph(figure 3(a-4)).

Through the structural analysis for the structured scale-free network, we point out that the intermediate region plays the crucial role for the evolution of cooperation. Hence, we add an intermediate node among the two hubs as shown in figure 3(b) to illustrate the effect of the intermediate region. We assume initially the node $\mathrm{x}$ on the intermediate region is a defector and others are the same as the figure 3(a-1). At figure $3(\mathrm{~b}-1), \pi\left(h_{l}\right)=N, \pi\left(h_{r}\right)=0$ and $\pi(x)=b$. If $x$ chooses $h_{l}$ to update its strategy, 


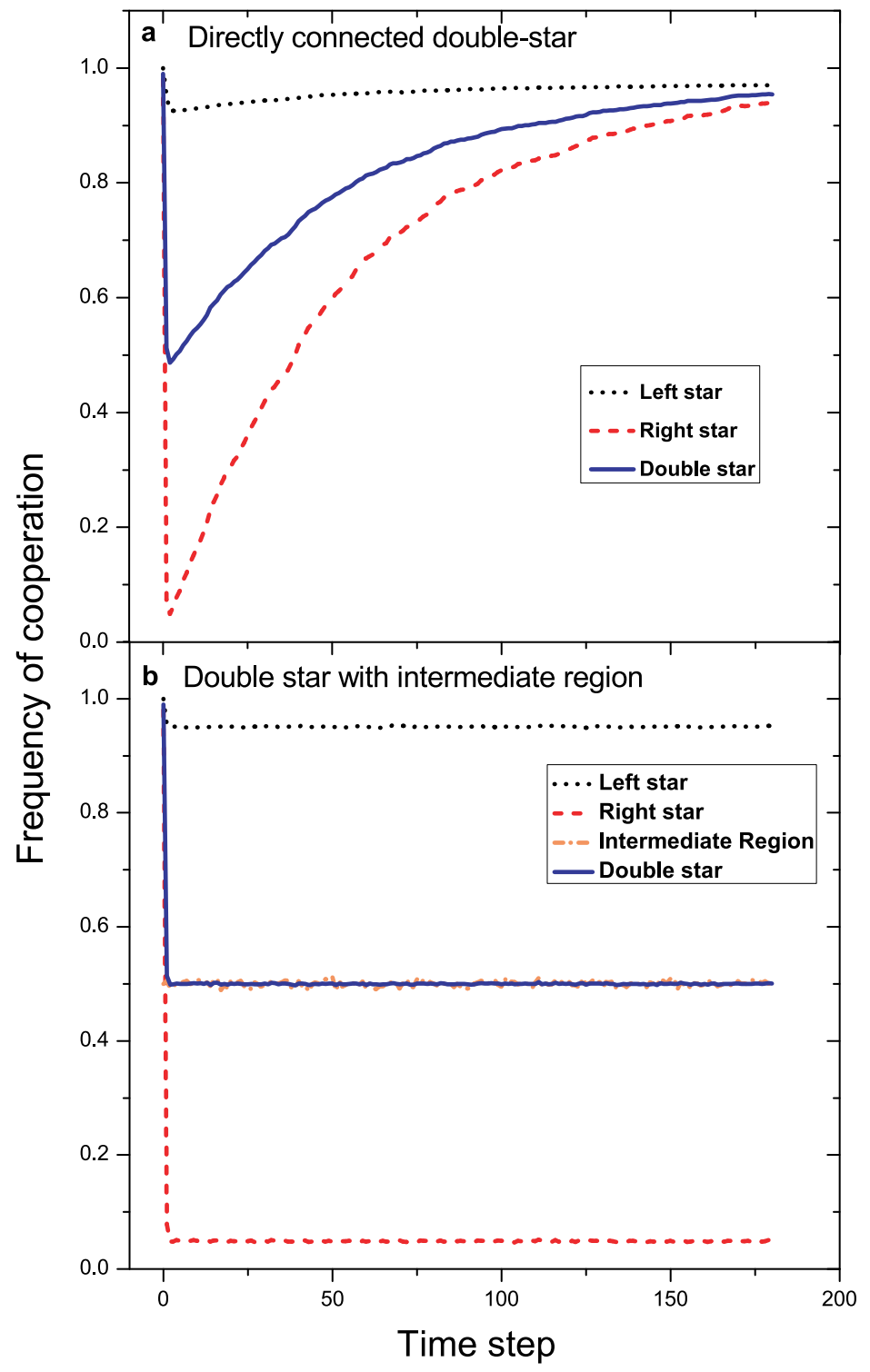

Figure 4. Evolution of cooperation under the PD game on the double-stars graphs. Initially the cooperator hub $h_{l}$ and the defector $h_{r}$ are linked with the same number of leaves, $N=M=50$. All leaves are cooperators. We fix $b=1.5$. The simulations are averaged over 1000 runs. (a) The frequency of cooperation $f_{c}$, on the left star (dot line) stays at nearly 1.0. $f_{c}$ in the right star (dashed line) first decreases to nearly 0 as defectors invades its leaves. Then at the lowest point, $h_{l}$ spreads the cooperation strategy to $h_{r}$ successfully. After that, the cooperators wipe up all the defectors on the right star. (b) We add a 10-node intermediate region between the double-star as shown in the right corner. Initially the nodes on the intermediate region randomly choose their strategies. The left star contains all cooperators (dot line). The $f_{c}$ on the right star (dash line) decreases to nearly 0 (but not 0 because of the random effect) dramatically and stands still. The $f_{c}$ of intermediate region (dash dot line) fluctuates around 0.5 as expected. The frequency of cooperation of the whole double star is shown as solid line. 
the probability for $x$ to turn into a cooperator is

$$
P_{4}=\frac{\pi\left(h_{l}\right)-\pi(x)}{b \max (2, N)}=\frac{N-b}{b N} .
$$

Also according to the above analysis, the defector hub will invade all its leaves, and then we get figure 3(b-3). At this point, $\pi(x)=1<\pi\left(h_{r}\right)=b$. It's impossible for $x$ to be invaded by the defector hub with probability

$$
P_{5}=\frac{\pi\left(h_{r}\right)-\pi(x)}{b \max (2, M)}=\frac{b-1}{b M} .
$$

Therefore, the defector hub will receive the benefit from $x$ when $x$ adopts the cooperative strategy, furthermore, it will invade $x$ and make $x$ become defector again. Then the process drops into a loop shown between figure 3(b-3) and figure 3(b-4), alternatively. The nodes on the intermediate region fluctuate their strategies and the defector hub is protected by the intermediate region against the invasion of cooperator hubs, resulting in the fact that the cooperative behavior is inhabited on the structured scale-free network for the PD game. In figure 3(b) we consider that the intermediate region only contains one node, and in the case of multiple nodes on the intermediate region the results do not change. Although we use the double-star graphs to illustrate the cooperation evolution on the scale-free networks, the analysis can heuristically reflect the microscopic organization of cooperation on the evolutionary dynamic of the PD game.

Furthermore, we have done extensive simulations shown in figure 4 to justify our above analysis. In the simulations we bring in a kind of random effect [22]. We assume that there are no direct connections among the leaves but adopt a strategy from each other with probability 0.1 which is the random strategy adoption. From figure 4 it is observed that for the double-star graph, the defector hubs can be invaded by the cooperator hub and after which, its neighbors also become cooperators again. Whereas, for the double-star graph with intermediate region, the leaves around the cooperative hub are cooperators and that around the defector hubs will hold on the defection strategies. However, the intermediate region will change their strategies under the influence of their neighbors. Hence, the cooperators' frequency of intermediate

region is 0.5. Figure 4 confirms our conclusions discussed above, i.e., the isolation of communication among hubs in the structured scale-free network decreases the emergence of cooperation.

\section{Conclusion}

In this paper, we have investigated the evolution of cooperation on the structured scale-free network for the PD game. In contrast to the conventional wisdom that the cooperation dominates on the BA scale-free network, the cooperative behavior is inhibited on the structured scale-free network. We find that neither the scale-free property nor the high clustering coefficient is the determinant factor for the inhibition 
of cooperation spreading on the structured scale-free network. Then what is the root cause? Comparing with the BA network, the age correlations are greatly suppressed on the structured scale-free network. In result, hubs are usually linked to low-degree nodes and simulations have confirmed the result that the node-distance among hubs are much larger on the structured scale-free network than on the BA network. Furthermore, to explain heuristically why the spread of cooperation is inhibited, we simplify the structured scale-free network as a double-star connected by an intermediate region, which is formed by low-degree nodes. Through detailed analysis we showed that the lack of age correlations and associated 'large-world' behavior are responsible for the inhibition of cooperation on the structured scale-free network.

\section{Acknowledgment}

This research was supported by the National Basic Research Program of China under grant No. 2007CB310806, the National Natural Science Foundation of China under Grant Nos. 60704044, 60873040 and 60873070, Shanghai Leading Academic Discipline Project No. B114, and the Program for New Century Excellent Talents in University of China (NCET-06-0376). Rong Zhihai acknowledges support from the National Science Foundation of P. R. China under Grant No. 60874089 and 70701009, the Shanghai Education Development Foundation under Grant No. 2008CG38.

\section{References}

[1] Axelrod R and Hamilton W D 1981 The evolution of cooperation Science 211, 1390-6.

[2] Nowak M A and May R M 1992 Evolutionary games and spatial chaos Nature 359, 826-9.

[3] Killingback T, Doebeli M and Knowlton N 1999 Variable investment, the continuous prisioner's dillemma and the origin of cooperation Proc. R. Soc. Lond. B 266, 1723-28.

[4] Hauert C and Doebeli M 2004 Spatial structure often inhibits the evolution of cooperation in the snowdrift game Nature 428, 643-46.

[5] Nowak M A and Sigmund K 2005 Evolution of indirect reciprocity Nature 437, 1291-8.

[6] Ohtsuki H, Hauert C, Lieberman E and Nowak M A 2006 A simple rule for the evoluation of cooperation on graphs and social networks Nature 441, 502-5.

[7] Hofbauer J and Sigmund K 1998 Evolutionary Games and Population Dynamics (Cambridge: Cambridge University Press).

[8] Maynard Smith J 1982 Evolution and the Theory of Games (Cambridge University Press, Cambridge, England).

[9] Gintis H 2000 Game Theory Evolving (Princeton University, Princeton, NJ).

[10] Axelrod R 1984 Evolution of Cooperation (Basic Books, New York).

[11] Kerr B, Riley M, Feldman M and Bohannan B J M 2002 Local dispersal and interaction promote coexistence in a real life game of rock-paper-scissors Nature 418, 171-4.

[12] Szabó G and Toke C 1998 Evolutionary prisoners dilemma game on a square lattice Phys. Rev. E 58, 69-73.

[13] Abramson G and Kuperman M 2001 Social games in a social network Phys. Rev. E 63, 030901(R).

[14] Faloutsos M, Faloutsos P and C. Faloutsos 1999 On power-law relationships of the Internet topology Comput. Commun. Rev 29, 251-62.

[15] Jeong H, Mason S P, Barabási A-L and Oltvai Z N 2001 Lethality and centrality in protein networks Nature 411, 41-2. 
[16] Newman M E J 2001 The structure of scientific collaboration networks Proc. Natl. Acad. Sci. USA 98, 404-9.

[17] Pastor-Satorras R and Vesignani A 2004 Evolution and Structure of the Internet: A Statistical Physics Approach (Cambridge: Cambridge University Press).

[18] Santos F C, Santos M D and Pacheco J M 2008 Social diversity promotes the emergence of cooperation in public goods games Nature 454, 213-7.

[19] Barabási A-L and Albert R 1999 Emergence of scaling in random networks Science 286, 509-12.

[20] Santos F C and Pacheco J M 2005 Scale-Free networks provide a unifying framework for the emergence of cooperation Phys. Rev. Lett. 95, 098104.

[21] Gómez-Gardeñes J, Campillo M, Floría L M and Moreno Y 2007 Dynamical organization of cooperation in complex topologies Phys. Rev. Lett. 98, 108103.

[22] Szabó G and Fáth G 2007 Evolutionary games on graphs Phys. Rep. 446, 97-216.

[23] Poncela J, Gómez-Gardeñes J, Floría L M and Moreno Y 2007 Robustness of cooperation in the evolutionary prisoner's dilemma on complex networks New J. Phys. 9, 184.

[24] Rong Z H, Li Xiang and Wang X F 2007 Roles of mixing patterns in cooperation on a scale-free networked game Phys. Rev. E 76, 027101.

[25] Wang W X, Lu J H, Chen G R and Hui P M 2008 Phase transition and hysteresis loop in structured games with global updating Phys. Rev. E 77, 046109.

[26] Klemm K and Eguíluz V M 2002 Highly clustered scale-free networks Phys. Rev. E 65, 036123.

[27] Barabási A-L and Albert R 2002 Statistical mechanics of complex networks Rev. Mod. Phys. 74, 47-97.

[28] Vázquez A, Boguñá M, Moreno Y, Pastor-Satorras R and Vespignani A 2003 Topology and correlations in structured scale-free networks Phys. Rev. E 67, 046111.

[29] Assenza S, Gómez-Gardeñes J and Latora V 2008 Enhancement of cooperation in highly clustered scale-free networks Phys. Rev. E 78, 017101.

[30] Holme P and Kim B J 2002 Growing scale-free networks with tunable clustering Phys. Rev. E 65, 026107.

[31] Eguíluz V M and Klemm K 2002 Epidemic threshold in structured scale-Free networks Phys. Rev. Lett. 89, 108701. 